\title{
ADAPTIVE FUZZY SLIDING MODE CONTROL OF UNCERTAIN NONLINEAR SYSTEMS
}

\author{
Wallace Moreira Bessa* \\ wmbessa@ufrnet.br \\ Roberto Souza Sá Barrêto ${ }^{\dagger}$ \\ bbetosab@gmail.com \\ *Universidade Federal do Rio Grande do Norte, Centro de Tecnologia, Departamento de Engenharia Mecânica, Campus \\ Universitário Lagoa Nova, CEP 59072-970, Natal, RN, Brazil \\ ${ }^{\dagger}$ Centro Federal de Educação Tecnológica Celso Suckow da Fonseca, Av. Maracanã 229, Bloco E, DEPES, CEP 20271-110, \\ Rio de Janeiro, RJ, Brazil
}

\begin{abstract}
This paper presents a detailed discussion about the convergence properties of a variable structure controller for uncertain single-input-single-output nonlinear systems (SISO). The adopted approach is based on the sliding mode control strategy and enhanced by an adaptive fuzzy algorithm to cope with modeling inaccuracies and external disturbances that can arise. The boundedness of all closed-loop signals and the convergence properties of the tracking error are analytically proven using Lyapunov's direct method and Barbalat's lemma. This result corrects flawed conclusions previously reached in the literature. An application of this adaptive fuzzy sliding mode controller to a second-order nonlinear system is also presented. The obtained numerical results demonstrate the improved control system performance.
\end{abstract}

KEYWORDS: Nonlinear Control, Sliding Modes, Fuzzy Logic, Adaptive Methods

\section{RESUMO}

Controle por Modos Deslizantes Nebuloso Adaptativo de Sistemas Incertos Não-lineares

Este trabalho apresenta uma discussão detalhada acerca das propriedades de convergência de um controlador à estrutura variável para sistemas incertos com uma entrada e uma saída

Artigo submetido em 28/11/2007 (Id.: 00831)

Revisado em 30/07/2008, 05/03/2009

Aceito sob recomendação do Editor Associado Prof. Takashi Yoneyama
(SISO). A abordagem adotada baseia-se na estratégia de controle por modos deslizantes e incorpora um algoritmo difuso adaptativo para compensar imprecisões de modelagem e perturbações externas que possam ocorrer. A limitação de todos os sinais do sistema em malha-fechada e as propriedades de convergência do erro de rastreamento são demonstradas analiticamente através do método direto de Liapunov e do lema de Barbalat. Este resultado corrige conclusões errôneas apresentadas anteriormente na literatura. Uma aplicação do controlador por modos deslizantes difuso adaptativo em um sistema não-linear de segunda ordem também é discutida. Os resultados obtidos numericamente confirmam o desempenho do controlador.

PALAVRAS-CHAVE: Controle Não-Linear, Modos Deslizantes, Lógica Difusa, Métodos Adaptativos

\section{INTRODUCTION}

Sliding mode control, due to its robustness against modeling imprecisions and external disturbances, has been successfully employed to nonlinear control problems. But a known drawback of conventional sliding mode controllers is the chattering effect. To overcome the undesired effects of the control chattering, Slotine (1984) proposed the adoption of a thin boundary layer neighboring the switching surface, by replacing the sign function by a saturation function. This substitution can minimize or, when desired, even completely eliminate chattering, but turns perfect tracking into a tracking with guaranteed precision problem, which actu- 
ally means that a steady-state error will always remain. In order to enhance the tracking performance inside the boundary layer, some adaptive strategy should be used for uncertainty/disturbance compensation.

Due to the possibility to express human experience in an algorithmic manner, fuzzy logic has been largely employed in the last decades to both control and identification of dynamical systems. In spite of the simplicity of this heuristic approach, in some situations a more rigorous mathematical treatment of the problem is required. Recently, much effort has been made to combine fuzzy logic with nonlinear control methodology. In (Wang, 1993) a globally stable adaptive fuzzy controller was proposed using Lyapunov stability theory to develop the adaptive law. Combining fuzzy logic with sliding mode control, Palm (1994) used the switching variable $s$ to define a fuzzy boundary layer. Some improvements to this control scheme appeared in (Chai and Tong, 1999) and (Berstecher et al., 2001). Wong et al. (2001) proposed a fuzzy logic controller which combines a sliding mode controller and a proportional plus integral controller. A sliding mode controller that incorporates a fuzzy tuning technique was analyzed in (Ha et al., 2001). By defining a generalized error transformation as a complement to the conventional switching variable, Liang and Su (2003) developed a stable fuzzy sliding mode control scheme. Cheng and Chien (2006) proposed an adaptive sliding mode controller based on T-S fuzzy models and Wu and Juang (2008) showed that fuzzy sliding surfaces can be established by solving a set of linear matrix inequalities.

A robust and very attractive approach was proposed in (Yoo and Ham, 1998). Yoo and Ham (1998) used fuzzy inference systems to approximate the unknown system dynamics within the sliding mode controller. Su et al. (2001), Wang et al. (2001), Chang et al. (2002) and also Kung and Chen (2005) suggested some improvements to this methodology. A drawback of this approach is the adoption of the state variables in the premise of the fuzzy rules. For higher-order systems the number of fuzzy sets and fuzzy rules becomes incredibly large, which compromises the applicability of this technique.

In this paper, an adaptive fuzzy sliding mode controller (AF$\mathrm{SMC}$ ) is proposed to deal with imprecise single-input-singleoutput (SISO) nonlinear systems. The proposed control scheme is based on (Yoo and Ham, 1998) but here an estimate of system dynamics is assumed to be known and the adaptive fuzzy inference system is adopted to compensate for modeling imprecisions and external disturbances. In order to reduce the number of fuzzy sets and rules and consequently simplify the design process, the switching variable $s$, instead of the state variables, is considered in the premise of the fuzzy rules. By replacing the sign function by the saturation function, the undesirable chattering effects are completely avoided. This control strategy has already been successfully applied to the dynamic positioning of remotely operated underwater vehicles (Bessa et al., 2008; Bessa, Dutra and Kreuzer, 2007) and to the chaos control in a nonlinear pendulum (Bessa, De Paula and Savi, 2007; De Paula et al., 2007). In this work, using Lyapunov's second method (also called Lyapunov's direct method) and Barbalat's lemma, the boundedness of all closed-loop signals and some convergence properties of the tracking error are analytically proven for an $n^{\text {th }}$ order uncertain SISO nonlinear system. This result also corrects a minor flaw in Slotine's work, by showing that the bounds of the error vector are different from the bounds provided in (Slotine, 1984). A simulation example is also presented in order to demonstrate that, when compared with a conventional sliding mode controller, the AFSMC shows an improved performance.

\section{ADAPTIVE FUZZY SLIDING MODE CONTROLLER}

Consider a class of $n^{\text {th }}$-order nonlinear systems:

$$
x^{(n)}=f(\mathbf{x})+b(\mathbf{x}) u+d
$$

where $u$ is the control input, the scalar variable $x$ is the output of interest, $x^{(n)}$ is the $n$-th time derivative of $x$, $\mathbf{x}=\left[x, \dot{x}, \ldots, x^{(n-1)}\right]$ is the system state vector, $d$ represents external disturbances and unmodeled dynamics, and $f, b: \mathbb{R}^{n} \rightarrow \mathbb{R}$ are both nonlinear functions.

In respect of the dynamic system presented in equation (1), the following assumptions will be made:

Assumption 1 The function $f$ is unknown but bounded by a known function of $\mathbf{x}$, i.e. $|\hat{f}(\mathbf{x})-f(\mathbf{x})| \leq F(\mathbf{x})$ where $\hat{f}$ is an estimate of $f$.

Assumption 2 The input gain $b$ is unknown but positive and bounded, i.e. $0<b_{\min } \leq b(\mathbf{x}) \leq b_{\max }$.

Assumption 3 The disturbance $d$ is unknown but bounded, i.e. $|d| \leq \delta$.

The proposed control problem is to ensure that, even in the presence of external disturbances and modeling imprecisions, the state vector $\mathbf{x}$ will follow a desired trajectory $\mathbf{x}_{d}=\left[x_{d}, \dot{x}_{d}, \ldots, x_{d}^{(n-1)}\right]$ in the state space.

Regarding the development of the control law the following assumptions should also be made:

Assumption 4 The state vector $\mathbf{x}$ is available. 
Assumption 5 The desired trajectory $\mathbf{x}_{d}$ is once differentiable in time. Furthermore, every element of vector $\mathbf{x}_{d}$, as well as $x_{d}^{(n)}$, is available and with known bounds.

Now, let $\tilde{x}=x-x_{d}$ be defined as the tracking error in the variable $x$, and

$$
\tilde{\mathbf{x}}=\mathbf{x}-\mathbf{x}_{d}=\left[\tilde{x}, \dot{\tilde{x}}, \ldots, \tilde{x}^{(n-1)}\right]
$$

as the tracking error vector.

Consider a sliding surface $S$ defined in the state space by the equation $s(\tilde{\mathbf{x}})=0$, with the function $s: \mathbb{R}^{n} \rightarrow \mathbb{R}$ satisfying

$$
s(\tilde{\mathbf{x}})=\left(\frac{d}{d t}+\lambda\right)^{n-1} \tilde{x}
$$

or conveniently rewritten as

$$
s(\tilde{\mathbf{x}})=\boldsymbol{\Lambda}^{\mathrm{T}} \tilde{\mathbf{x}}
$$

where $\boldsymbol{\Lambda}=\left[c_{n-1} \lambda^{n-1}, \ldots, c_{1} \lambda, c_{0}\right]$ and $c_{i}$ states for binomial coefficients, i.e.

$c_{i}=\left(\begin{array}{c}n-1 \\ i\end{array}\right)=\frac{(n-1) !}{(n-i-1) ! i !}, \quad i=0,1, \ldots, n-1$

which makes $c_{n-1} \lambda^{n-1}+\cdots+c_{1} \lambda+c_{0}$ a Hurwitz polynomial.

From equation 4 , it can be easily verified that $c_{0}=1$, for $\forall n \geq 1$. Thus, for notational convenience, the time derivative of $s$ will be written in the following form:

$$
\dot{s}=\boldsymbol{\Lambda}^{\mathrm{T}} \dot{\tilde{\mathbf{x}}}=\tilde{x}^{(n)}+\boldsymbol{\Lambda}_{u}^{\mathrm{T}} \tilde{\mathbf{x}}
$$

where $\boldsymbol{\Lambda}_{u}=\left[0, c_{n-1} \lambda^{n-1}, \ldots, c_{1} \lambda\right]$.

Now, let the problem of controlling the uncertain nonlinear system (1) be treated in a Filippov's way (Filippov, 1988), defining a control law composed by an equivalent control $\hat{u}=\hat{b}^{-1}\left(-\hat{f}-\hat{d}+x_{d}^{(n)}-\boldsymbol{\Lambda}_{u}^{\mathrm{T}} \tilde{\mathbf{x}}\right)$ and a discontinuous term $-K \operatorname{sgn}(s)$ :

$$
u=\hat{b}^{-1}\left(-\hat{f}-\hat{d}+x_{d}^{(n)}-\boldsymbol{\Lambda}_{u}^{\mathrm{T}} \tilde{\mathbf{x}}\right)-K \operatorname{sgn}(s)
$$

where $\hat{d}$ is an estimate of $d, \hat{b}=\sqrt{b_{\max } b_{\min }}$ is an estimate of $b, K$ is a positive gain and $\operatorname{sgn}(\cdot)$ is defined as

$$
\operatorname{sgn}(s)=\left\{\begin{array}{rll}
-1 & \text { if } & s<0 \\
0 & \text { if } & s=0 \\
1 & \text { if } & s>0
\end{array}\right.
$$

Based on Assumptions $1-3$ and considering that $\beta^{-1} \leq$ $\hat{b} / b \leq \beta$, where $\beta=\sqrt{b_{\max } / b_{\min }}$, the gain $K$ should be chosen according to

$$
K \geq \beta \hat{b}^{-1}(\eta+\delta+|\hat{d}|+F)+(\beta-1)|\hat{u}|
$$

where $\eta$ is a strictly positive constant related to the reaching time.

Based on the sliding mode methodology (Slotine and Li, 1991), it can be easily verified that (6) is sufficient to impose the sliding condition:

$$
\begin{aligned}
\frac{1}{2} \frac{d}{d t} s^{2}= & s \dot{s}=\left(\tilde{x}^{(n)}+\boldsymbol{\Lambda}_{u}^{\mathrm{T}} \tilde{\mathbf{x}}\right) s=\left(x^{(n)}-x_{d}^{(n)}+\boldsymbol{\Lambda}_{u}^{\mathrm{T}} \tilde{\mathbf{x}}\right) s \\
= & \left(f+b u+d-x_{d}^{(n)}+\boldsymbol{\Lambda}_{u}^{\mathrm{T}} \tilde{\mathbf{x}}\right) s \\
= & {\left[f+b \hat{b}^{-1}\left(-\hat{f}-\hat{d}+x_{d}^{(n)}-\boldsymbol{\Lambda}_{u}^{\mathrm{T}} \tilde{\mathbf{x}}\right)+\right.} \\
& \left.\quad-b K \operatorname{sgn}(s)+d-\left(x_{d}^{(n)}-\boldsymbol{\Lambda}_{u}^{\mathrm{T}} \tilde{\mathbf{x}}\right)\right] s
\end{aligned}
$$

Recalling that $\hat{u}=\hat{b}^{-1}\left(-\hat{f}-\hat{d}+x_{d}^{(n)}-\boldsymbol{\Lambda}_{u}^{\mathrm{T}} \tilde{\mathbf{x}}\right)$, and noting that $f=\hat{f}-(\hat{f}-f)$ and $d=\hat{d}-(\hat{d}-d)$, one has

$$
\frac{1}{2} \frac{d}{d t} s^{2}=-[(\hat{f}-f)+(\hat{d}-d)+\hat{b} \hat{u}-b \hat{u}+b K \operatorname{sgn}(s)] s
$$

Thus, considering assumptions $1-3$ and defining $K$ according to (8), it follows that

$$
\frac{1}{2} \frac{d}{d t} s^{2}=s \dot{s} \leq-\eta|s|
$$

Then, dividing by $|s|$ and integrating both sides over the interval $0 \leq t \leq t_{s}$, where $t_{s}$ is the time required to hit $S$, gives

$$
\begin{gathered}
\int_{0}^{t_{s}} \frac{s}{|s|} \dot{s} d t \leq-\int_{0}^{t_{s}} \eta d t \\
\left|s\left(t=t_{s}\right)\right|-|s(t=0)| \leq-\eta t_{s}
\end{gathered}
$$

In this way, noting that $\left|s\left(t=t_{s}\right)\right|=0$, one has 


$$
t_{s} \leq \frac{|s(t=0)|}{\eta}
$$

and, consequently, the finite time convergence to the sliding surface $S$.

In order to obtain a good approximation to the disturbance $d$, the estimate $\hat{d}$ will be computed directly by an adaptive fuzzy algorithm.

The adopted fuzzy inference system was the zero order TSK (Takagi-Sugeno-Kang) (Jang et al., 1997), whose rules can be stated in a linguistic manner as follows:

$$
\text { If } s \text { is } S_{r} \text { then } \hat{d}_{r}=\hat{D}_{r} ; r=1,2, \ldots, N
$$

where $S_{r}$ are fuzzy sets, whose membership functions could be properly chosen, and $\hat{D}_{r}$ is the output value of each one of the $N$ fuzzy rules.

At this point, it should be highlighted that the adoption of the switching variable $s$ in the premise of the rules, instead of the state variables as in (Yoo and Ham, 1998; Su et al., 2001; Wang et al., 2001; Chang et al., 2002; Kung and Chen, 2005), leads to a smaller number of fuzzy sets and rules, which simplifies the design process. Considering that external disturbances are independent of the state variables, the choice of a combined tracking error measure $s$ also seems to be more appropriate in this case.

Considering that each rule defines a numerical value as output $\hat{D}_{r}$, the final output $\hat{d}$ can be computed by a weighted average:

$$
\hat{d}(s)=\frac{\sum_{r=1}^{N} w_{r} \cdot \hat{d}_{r}}{\sum_{r=1}^{N} w_{r}}
$$

or, similarly,

$$
\hat{d}(s)=\hat{\mathbf{D}}^{\mathrm{T}} \mathbf{\Psi}(s)
$$

where, $\hat{\mathbf{D}}=\left[\hat{D}_{1}, \hat{D}_{2}, \ldots, \hat{D}_{N}\right]$ is the vector containing the attributed values $\hat{D}_{r}$ to each rule $r, \boldsymbol{\Psi}(s)=$ $\left[\psi_{1}(s), \psi_{2}(s), \ldots, \psi_{N}(s)\right]$ is a vector with components $\psi_{r}(s)=w_{r} / \sum_{r=1}^{N} w_{r}$ and $w_{r}$ is the firing strength of each rule.

To ensure the best possible estimate $\hat{d}(s)$ to the disturbance $d$, the vector of adjustable parameters can be automatically updated by the following adaptation law:

$$
\dot{\hat{\mathbf{D}}}=\varphi s \Psi(s)
$$

where $\varphi$ is a strictly positive constant related to the adaptation rate.

Equation (11) also shows that there is no adaptation when states are on the sliding surface, $\dot{\hat{\mathbf{D}}}=0$ for $s=0$.

It's important to emphasize that the chosen adaptation law, equation (11), must not only provide a good approximation to disturbance $d$ but also not compromise the attractiveness of the sliding surface, as will be proven in the following theorem.

Theorem 1 Consider the uncertain nonlinear system (1) and assumptions 1-5. Then, the controller defined by (6), (8), (10) and (11) ensures the convergence of the tracking error vector to the sliding surface $S$.

Proof: Let a positive-definite function $V_{1}$ be defined as

$$
V_{1}(t)=\frac{1}{2} s^{2}+\frac{1}{2 \varphi} \boldsymbol{\Delta}^{\mathrm{T}} \boldsymbol{\Delta}
$$

where $\boldsymbol{\Delta}=\hat{\mathbf{D}}-\hat{\mathbf{D}}^{*}$ and $\hat{\mathbf{D}}^{*}$ is the optimal parameter vector, associated to the optimal estimate $\hat{d}^{*}(s)$. Thus, the time derivative of $V_{1}$ is

$$
\begin{aligned}
\dot{V}_{1}(t) & =s \dot{s}+\varphi^{-1} \boldsymbol{\Delta}^{\mathrm{T}} \dot{\boldsymbol{\Delta}} \\
& =\left(\tilde{x}^{(n)}+\boldsymbol{\Lambda}_{u}^{\mathrm{T}} \tilde{\mathbf{x}}\right) s+\varphi^{-1} \boldsymbol{\Delta}^{\mathrm{T}} \dot{\boldsymbol{\Delta}} \\
& =\left(x^{(n)}-x_{d}^{(n)}+\boldsymbol{\Lambda}_{u}^{\mathrm{T}} \tilde{\mathbf{x}}\right) s+\varphi^{-1} \boldsymbol{\Delta}^{\mathrm{T}} \dot{\boldsymbol{\Delta}} \\
& =\left(f+b u+d-x_{d}^{(n)}+\boldsymbol{\Lambda}_{u}^{\mathrm{T}} \tilde{\mathbf{x}}\right) s+\varphi^{-1} \boldsymbol{\Delta}^{\mathrm{T}} \dot{\boldsymbol{\Delta}} \\
& =\left[f+b \hat{b}^{-1}\left(-\hat{f}-\hat{d}+x_{d}^{(n)}-\boldsymbol{\Lambda}_{u}^{\mathrm{T}} \tilde{\mathbf{x}}\right)+\right. \\
& \left.-b K \operatorname{sgn}(s)+d-\left(x_{d}^{(n)}-\boldsymbol{\Lambda}_{u}^{\mathrm{T}} \tilde{\mathbf{x}}\right)\right] s+\varphi^{-1} \boldsymbol{\Delta}^{\mathrm{T}} \dot{\boldsymbol{\Delta}}
\end{aligned}
$$

Defining the minimum approximation error as $\varepsilon=\hat{d}^{*}(s)-d$, recalling that $\hat{u}=\hat{b}^{-1}\left(-\hat{f}-\hat{d}+x_{d}^{(n)}-\boldsymbol{\Lambda}_{u}^{\mathrm{T}} \tilde{\mathbf{x}}\right)$, and noting that $\dot{\boldsymbol{\Delta}}=\dot{\hat{\mathbf{D}}}, f=\hat{f}-(\hat{f}-f)$ and $d=\hat{d}-(\hat{d}-d), \dot{V}_{1}$ becomes: 


$$
\begin{aligned}
\dot{V}_{1}(t) & =-\left[(\hat{f}-f)+\varepsilon+\left(\hat{d}-\hat{d}^{*}\right)+\hat{b} \hat{u}-b \hat{u}+\right. \\
& +b K \operatorname{sgn}(s)] s+\varphi^{-1} \boldsymbol{\Delta}^{\mathrm{T}} \dot{\hat{\mathbf{D}}} \\
& =-\left[(\hat{f}-f)+\varepsilon+\left(\hat{\mathbf{D}}-\hat{\mathbf{D}}^{*}\right)^{\mathrm{T}} \boldsymbol{\Psi}(s)+\hat{b} \hat{u}-b \hat{u}+\right. \\
& +b K \operatorname{sgn}(s)] s+\varphi^{-1} \boldsymbol{\Delta}^{\mathrm{T}} \dot{\hat{\mathbf{D}}} \\
& =-[(\hat{f}-f)+\varepsilon+\hat{b} \hat{u}-b \hat{u}+b K \operatorname{sgn}(s)] s+ \\
& +\varphi^{-1} \boldsymbol{\Delta}^{\mathrm{T}}(\dot{\hat{\mathbf{D}}}-\varphi s \boldsymbol{\Psi}(s))
\end{aligned}
$$

Thus, by applying the adaptation law (11) to $\dot{\hat{\mathbf{D}}}$ :

$$
\dot{V}_{1}(t)=-[(\hat{f}-f)+\varepsilon+\hat{b} \hat{u}-b \hat{u}+b K \operatorname{sgn}(s)] s
$$

Furthermore, considering assumptions $1-3$, defining $K$ according to (8) and verifying that $|\varepsilon|=\left|\hat{d}^{*}-d\right| \leq|\hat{d}-d| \leq$ $|\hat{d}|+\delta$, it follows

$$
\dot{V}_{1}(t) \leq-\eta|s|
$$

which implies $V_{1}(t) \leq V_{1}(0)$ and that $s$ and $\boldsymbol{\Delta}$ are bounded. Considering that $s(\tilde{\mathbf{x}})=\boldsymbol{\Lambda}^{\mathrm{T}} \tilde{\mathbf{x}}$, it can be verified that $\tilde{\mathbf{x}}$ is also bounded. Hence, equation (5) and Assumption 5 implies that $\dot{s}$ is also bounded.

Integrating both sides of (12) shows that

$$
\lim _{t \rightarrow \infty} \int_{0}^{t} \eta|s| d \tau \leq \lim _{t \rightarrow \infty}\left[V_{1}(0)-V_{1}(t)\right] \leq V_{1}(0)<\infty
$$

Since the absolute value function is uniformly continuous, it follows from Barbalat's lemma (Khalil, 2001) that $s \rightarrow 0$ as $t \rightarrow \infty$, which ensures the convergence of the tracking error vector to the sliding surface $S$.

Remark 1 Although Theorem 1 only guarantees the asymptotic convergence to $S$, the control law (6) actually ensures the finite time convergence to $S$, as previously verified by imposing the sliding condition to system states, and, consequently, the exponential stability of the closed-loop system.

In spite of the demonstrated properties of the controller, the presence of a discontinuous term in the control law leads to the well known chattering effect. In order to avoid these undesirable high-frequency oscillations of the controlled variable, the sign function can be replaced by a saturation function (Slotine, 1984), defined as:

$$
\operatorname{sat}(s / \phi)=\left\{\begin{array}{ccc}
\operatorname{sgn}(s) & \text { if } & |s / \phi| \geq 1 \\
s / \phi & \text { if } & |s / \phi|<1
\end{array}\right.
$$

This substitution smoothes out the control discontinuity and introduces a thin boundary layer, $S_{\phi}$, in the neighborhood of the switching surface

$$
S_{\phi}=\left\{\tilde{\mathbf{x}} \in \mathbb{R}^{n}|| s(\tilde{\mathbf{x}}) \mid \leq \phi\right\}
$$

where $\phi$ is a strictly positive constant that represents the boundary layer thickness.

Thus, the resulting control law can be stated as follows

$$
u=\hat{b}^{-1}\left(-\hat{f}-\hat{d}+x_{d}^{(n)}-\boldsymbol{\Lambda}_{u}^{\mathrm{T}} \tilde{\mathbf{x}}\right)-K \operatorname{sat}\left(\frac{s}{\phi}\right)
$$

The proof of the boundedness of all closed-loop signals relies on the following lemma:

Lemma 2 Let the boundary layer be defined as $S_{\phi}=\{\tilde{\mathbf{x}} \in$ $\left.\mathbb{R}^{n}|| s(\tilde{\mathbf{x}}) \mid \leq \phi\right\}$, then for all trajectories starting inside $S_{\phi}$, the tracking error vector will exponentially converge to a closed region $\Phi=\left\{\tilde{\mathbf{x}} \in \mathbb{R}^{n}|| \tilde{x}^{(i)} \mid \leq \zeta_{i} \lambda^{i-n+1} \phi, i=\right.$ $0,1, \ldots, n-1\}$, with $\zeta_{i}$ defined according to

$$
\zeta_{i}=\left\{\begin{array}{cll}
1 & \text { for } & i=0 \\
1+\sum_{j=0}^{i-1}\left(\begin{array}{l}
i \\
j
\end{array}\right) \zeta_{j} & \text { for } & i=1,2, \ldots, n-1 .
\end{array}\right.
$$

Proof: From the definition of $s$, equation (3), and considering that $|s(\mathbf{x})| \leq \phi$ may be rewritten as $-\phi \leq s(\mathbf{x}) \leq \phi$, one has

$$
-\phi \leq c_{0} \tilde{x}^{(n-1)}+c_{1} \lambda \tilde{x}^{(n-2)}+\cdots+c_{n-2} \lambda^{n-2} \dot{\tilde{x}}+c_{n-1} \lambda^{n-1} \tilde{x} \leq \phi
$$

Multiplying (16) by $e^{\lambda t}$ yields

$$
-\phi e^{\lambda t} \leq \frac{d^{n-1}}{d t^{n-1}}\left(\tilde{x} e^{\lambda t}\right) \leq \phi e^{\lambda t}
$$

Thus, integrating (17) $n-1$ times between 0 and $t$ gives 


$$
\begin{gathered}
-\frac{\phi}{\lambda^{n-1}} e^{\lambda t}+\left(\left.\frac{d^{n-2}}{d t^{n-2}}\left(\tilde{x} e^{\lambda t}\right)\right|_{t=0}+\frac{\phi}{\lambda}\right) \frac{t^{n-2}}{(n-2) !}+\cdots+ \\
+\left(\tilde{x}(0)+\frac{\phi}{\lambda^{n-1}}\right) \leq \tilde{x} e^{\lambda t} \leq \frac{\phi}{\lambda^{n-1}} e^{\lambda t}+ \\
+\left(\left.\frac{d^{n-2}}{d t^{n-2}}\left(\tilde{x} e^{\lambda t}\right)\right|_{t=0}+\frac{\phi}{\lambda}\right) \frac{t^{n-2}}{(n-2) !}+\cdots+\left(\tilde{x}(0)+\frac{\phi}{\lambda^{n-1}}\right)
\end{gathered}
$$

Furthermore, dividing (18) by $e^{\lambda t}$, it can be easily verified that, for $t \rightarrow \infty$,

$$
-\frac{\phi}{\lambda^{n-1}} \leq \tilde{x}(t) \leq \frac{\phi}{\lambda^{n-1}}
$$

Considering the $(n-2)^{\text {th }}$ integral of (17)

$$
\begin{gathered}
-\frac{\phi}{\lambda^{n-2}} e^{\lambda t}-\left(\left|\frac{d^{n-2}}{d t^{n-2}}\left(\tilde{x} e^{\lambda t}\right)\right|_{t=0}+\frac{\phi}{\lambda}\right) \frac{t^{n-3}}{(n-3) !}-\cdots \\
-\left(|\dot{\tilde{x}}(0)|+\frac{\phi}{\lambda^{n-2}}\right) \leq \frac{d}{d t}\left(\tilde{x} e^{\lambda t}\right) \leq \frac{\phi}{\lambda^{n-2}} e^{\lambda t}+ \\
\left(\left|\frac{d^{n-2}}{d t^{n-2}}\left(\tilde{x} e^{\lambda t}\right)\right|_{t=0}+\frac{\phi}{\lambda}\right) \frac{t^{n-3}}{(n-3) !}+\cdots+\left(|\dot{\tilde{x}}(0)|+\frac{\phi}{\lambda^{n-2}}\right)
\end{gathered}
$$

and noting that $d\left(\tilde{x} e^{\lambda t}\right) / d t=\dot{\tilde{x}} e^{\lambda t}+\tilde{x} \lambda e^{\lambda t}$, by imposing the bounds (19) to (20) and dividing again by $e^{\lambda t}$, it follows that, for $t \rightarrow \infty$,

$$
-2 \frac{\phi}{\lambda^{n-2}} \leq \dot{\tilde{x}}(t) \leq 2 \frac{\phi}{\lambda^{n-2}}
$$

Now, applying the bounds (19) and (21) to the $(n-3)^{\text {th }}$ integral of (17) and dividing once again by $e^{\lambda t}$, it follows that, for $t \rightarrow \infty$,

$$
-6 \frac{\phi}{\lambda^{n-3}} \leq \ddot{\tilde{x}}(t) \leq 6 \frac{\phi}{\lambda^{n-3}}
$$

The same procedure can be successively repeated until the bounds for $\tilde{x}^{(n-1)}$ are achieved:

$$
\begin{aligned}
-\left(1+\sum_{i=0}^{n-2}\left(\begin{array}{c}
n-1 \\
i
\end{array}\right) \zeta_{i}\right) \phi \leq \tilde{x}^{(n-1)} \leq \\
\left(1+\sum_{i=0}^{n-2}\left(\begin{array}{c}
n-1 \\
i
\end{array}\right) \zeta_{i}\right) \phi
\end{aligned}
$$

where the coefficients $\zeta_{i}(i=0,1, \ldots, n-2)$ are related to the previously obtained bounds of each $\tilde{x}^{(i)}$ and can be summarized as in (15).

In this way, by inspection of the integrals of (17), as well as (19), (21), (22), (23) and the other omitted bounds, it follows that the tracking error vector will exponentially converge to a closed region $\Phi=\left\{\tilde{\mathbf{x}} \in \mathbb{R}^{n}|| \tilde{x}^{(i)} \mid \leq \zeta_{i} \lambda^{i-n+1} \phi, i=\right.$ $0,1, \ldots, n-1\}$.

Remark 2 Lemma 2 corrects a minor error in (Slotine, 1984). Slotine proposed that the bounds for $\tilde{x}^{(i)}$ could be summarized as $\left|\tilde{x}^{(i)}\right| \leq 2^{i} \lambda^{i-n+1} \phi, i=0,1, \ldots, n-1$. Although both results lead to same bounds for $\tilde{x}$ and $\dot{\tilde{x}}$, they start to differ from each other when the order of the derivative is higher than one, $i>1$. For example, according to Slotine the bounds for the second derivative would be $|\ddot{\tilde{x}}| \leq 4 \phi \lambda^{3-n}$ and not $|\ddot{\tilde{x}}| \leq 6 \phi \lambda^{3-n}$, as demonstrated in Lemma 2 .

The mistake in Slotine's work concerns the numeric coefficient in the tracking error bounds. Slotine (1984) did not considered the numeric value of the previously obtained bounds to compute the bounds of $\tilde{x}^{(i)}$. For example, if the coefficient 2 in $|\dot{\tilde{x}}| \leq 2 \phi / \lambda^{n-2}$ was not take into account to estimate the bounds of $\ddot{\tilde{x}}$, Lemma 2 would also lead to same erroneous result. In (Slotine, 1984) this error also occurs with the bounds of every derivative whose order is higher than one, $i>1$. Although the bounds proposed by Slotine (1984) are incorrect, they are until now widely evoked to establish the boundedness and convergence properties of many control schemes (Sharaf-Eldin et al., 1999; Zhang and Panda, 1999; Liang and Su, 2003; Wang et al., 2004; Chen et al., 2005; Wang and $\mathrm{Su}, 2006$; Zhang and Yi, 2007).

Finally, the boundedness and convergence properties of the tracking error are established in Theorem 3.

Theorem 3 Consider the uncertain nonlinear system (1) and assumptions 1-5. Then, the controller defined by (14), (8), (10) and (11) ensures the finite-time convergence of tracking error vector the to the boundary layer and its exponential convergence to the closed region $\Phi=\{\tilde{\mathbf{x}} \in$ $\left.\mathbb{R}^{n}|| \tilde{x}^{(i)} \mid \leq \zeta_{i} \lambda^{i-n+1} \phi, i=0,1, \ldots, n-1\right\}$.

Proof: Let a positive-definite Lyapunov function candidate $V_{2}$ be defined as

$$
V_{2}(t)=\frac{1}{2} s_{\phi}^{2}
$$

where $s_{\phi}$ is a measure of the distance of the current state to the boundary layer, and can be computed as follows 


$$
s_{\phi}=s-\phi \operatorname{sat}\left(\frac{s}{\phi}\right)
$$

Noting that $s_{\phi}=0$ inside the boundary layer and $\dot{s}_{\phi}=\dot{s}$, we get $\dot{V}_{2}(t)=0$ inside $S_{\phi}$, and outside

$$
\begin{aligned}
\dot{V}_{2}(t) & =s_{\phi} \dot{s}_{\phi}=s_{\phi} \dot{s}=\left(x^{(n)}-x_{d}^{(n)}+\boldsymbol{\Lambda}_{u}^{\mathrm{T}} \tilde{\mathbf{x}}\right) s_{\phi} \\
& =\left(f+b u+d-x_{d}^{(n)}+\boldsymbol{\Lambda}_{u}^{\mathrm{T}} \tilde{\mathbf{x}}\right) s_{\phi}
\end{aligned}
$$

It can be easily verified that outside the boundary layer the control law (14) takes the following form:

$$
u=\hat{b}^{-1}\left(-\hat{f}-\hat{d}+x_{d}^{(n)}-\boldsymbol{\Lambda}_{u}^{\mathrm{T}} \tilde{\mathbf{x}}\right)-K \operatorname{sgn}\left(s_{\phi}\right)
$$

Thus, the time derivative $\dot{V}_{2}$ can be written as

$$
\begin{array}{r}
\dot{V}_{2}(t)=\left[f+b \hat{b}^{-1}\left(-\hat{f}-\hat{d}+x_{d}^{(n)}-\boldsymbol{\Lambda}_{u}^{\mathrm{T}} \tilde{\mathbf{x}}\right)-b K \operatorname{sgn}\left(s_{\phi}\right)+\right. \\
\left.+d-\left(x_{d}^{(n)}-\boldsymbol{\Lambda}_{u}^{\mathrm{T}} \tilde{\mathbf{x}}\right)\right] s_{\phi}
\end{array}
$$

Recalling that $\hat{u}=\hat{b}^{-1}\left(-\hat{f}-\hat{d}+x_{d}^{(n)}-\boldsymbol{\Lambda}_{u}^{\mathrm{T}} \tilde{\mathbf{x}}\right)$, and noting that $f=\hat{f}-(\hat{f}-f)$ and $d=\hat{d}-(\hat{d}-d)$, one has

$$
\dot{V}_{2}(t)=-\left[(\hat{f}-f)+(\hat{d}-d)+\hat{b} \hat{u}-b \hat{u}+b K \operatorname{sgn}\left(s_{\phi}\right)\right] s_{\phi}
$$

So, considering Assumptions $1-3$ and defining $K$ according to $(8), \dot{V}_{2}$ becomes:

$$
\dot{V}_{2}(t) \leq-\eta\left|s_{\phi}\right|
$$

which implies $V_{2}(t) \leq V_{2}(0)$ and that $s_{\phi}$ is bounded. From the definition of $s_{\phi}$, it can be easily verified that $s$ is bounded. Considering that $s(\tilde{\mathbf{x}})=\boldsymbol{\Lambda}^{\mathrm{T}} \tilde{\mathbf{x}}$, it can be verified that $\tilde{\mathbf{X}}$ is also bounded. Hence, equation (5) and Assumption 5 implies that $\dot{s}$ is also bounded.

The finite-time convergence of the states to the boundary layer can be shown by recalling that

$$
\dot{V}_{2}(t)=\frac{1}{2} \frac{d}{d t} s_{\phi}^{2}=s_{\phi} \dot{s}_{\phi} \leq-\eta\left|s_{\phi}\right|
$$

Then, dividing by $\left|s_{\phi}\right|$ and integrating both sides over the interval $0 \leq t \leq t_{\phi}$, where $t_{\phi}$ is the time required to hit $S_{\phi}$, gives

$$
\begin{gathered}
\int_{0}^{t_{\phi}} \frac{s_{\phi}}{\left|s_{\phi}\right|} \dot{s}_{\phi} d t \leq-\int_{0}^{t_{\phi}} \eta d t \\
\left|s_{\phi}\left(t=t_{\phi}\right)\right|-\left|s_{\phi}(t=0)\right| \leq-\eta t_{\phi}
\end{gathered}
$$

In this way, noting that $\left|s_{\phi}\left(t=t_{\phi}\right)\right|=0$, one has

$$
t_{\phi} \leq \frac{\left|s_{\phi}(t=0)\right|}{\eta}
$$

which guarantees the attractiveness of the boundary layer. Thus, it follows from Lemma 2 that, for $t \geq 0$, states will exponentially converge to the closed region $\Phi$. This ensures the boundedness of all closed-loop signals and completes the proof.

\section{ILLUSTRATIVE EXAMPLE}

To demonstrate the improved performance of the adaptive fuzzy sliding mode controller (AFSMC) over the conventional sliding mode controller (SMC), consider a damped Duffing equation subjected to an external disturbance $d$

$$
\ddot{x}+0.2 \dot{x}+x^{3}-x=u+d
$$

According to the previously described scheme, the control law should be chosen as follows

$$
u=0.2 \dot{x}+x^{3}-x-\hat{d}+\ddot{x}_{d}-\lambda \dot{\tilde{x}}-K \operatorname{sat}\left(\frac{s}{\phi}\right)
$$

with $K=\eta+\delta+|\hat{d}|$ and, for a second order system, $s=$ $\dot{\tilde{x}}+\lambda \tilde{x}$.

The simulation studies were performed with an implementation in $\mathrm{C}$, with sampling rates of $500 \mathrm{~Hz}$ for control system and $1 \mathrm{kHz}$ for the Duffing oscillator. The differential equations of the dynamic model were numerically solved with a fourth order Runge-Kutta method. The disturbance was chosen as $d=0.3 \sin (0.4 \pi t)$ and the other used parameters were $\delta=0.3, \eta=0.1, \lambda=0.6, \phi=0.02$ and $\gamma=40$. Concerning the fuzzy system, triangular and trapezoidal membership functions were adopted for $S_{r}$, with the central values defined as shown in Fig. 1. It is also important to emphasize, that the vector of adjustable parameters was initialized with zero values, $\hat{\mathbf{D}}=\mathbf{0}$, and updated at each iteration step according to the adaptation law, equation (11). 


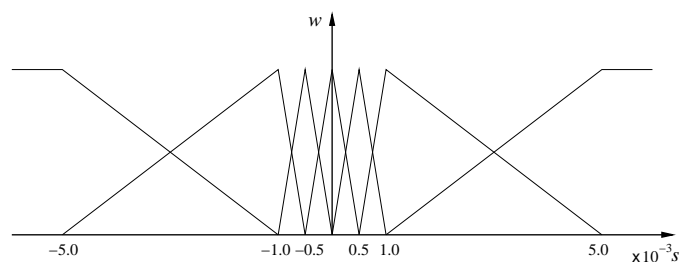

Figure 1: Adopted fuzzy membership functions.

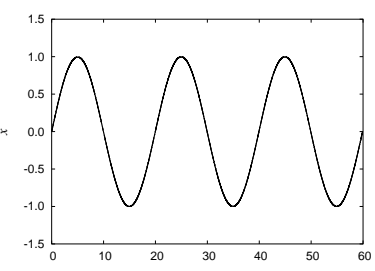

(a) Tracking performance

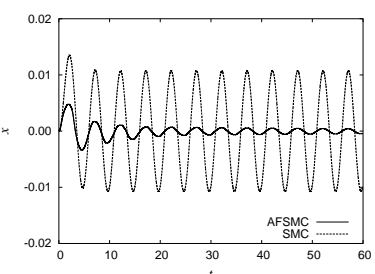

(c) Tracking error

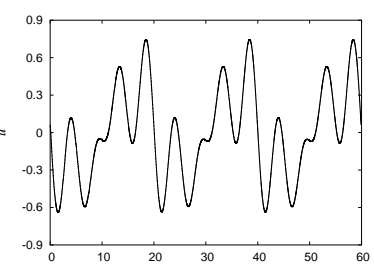

(b) Control action.

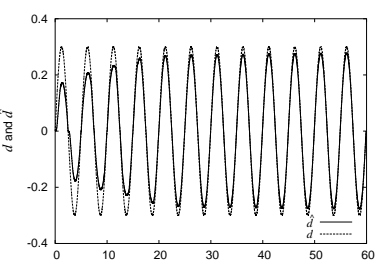

(d) Convergence of $\hat{d}$ to $d$.
Figure 2: Tracking of $x_{d}=\sin (0.1 \pi t)$ with $\tilde{\mathbf{x}}(0)=\mathbf{0}$.

In order to evaluate the control system performance, two different numerical simulations were performed. In the first case, it was considered that the initial state coincides with the initial desired state, $\tilde{\mathbf{x}}(0)=[\tilde{x}(0), \dot{\tilde{x}}(0)]=\mathbf{0}$. Fig. 2 gives the corresponding results for the tracking of $x_{d}=$ $\sin (0.1 \pi t)$.

As observed in Fig. 2, even in the presence of external disturbances, the adaptive fuzzy sliding mode controller (AFSMC) is capable to provide the trajectory tracking with a small associated error and no chattering at all. It can be also verified that the proposed control law provides a smaller tracking error when compared with the conventional sliding mode controller (SMC), Fig. 2(c). The improved performance of AFSMC over SMC is due to its ability to recognize and compensate the external disturbances, Fig. 2(d). For purpose of simulation, the AFSMC can be easily converted to the classical SMC by setting the adaptation rate to zero, $\varphi=0$.

In the second simulation study, the initial state and initial desired state are not equal, $\tilde{\mathbf{x}}(0)=[-0.7,-0.1]$. The chosen parameters, as well as the disturbance and the desired trajectory, were defined as before. Fig. 3 shows the obtained results.

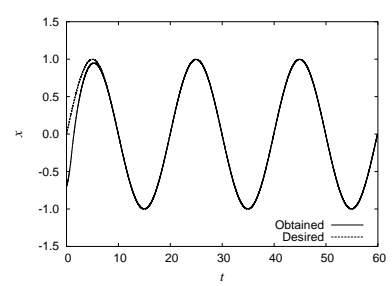

(a) Tracking performance.

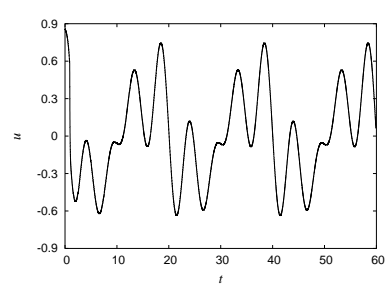

(b) Control variable.
Figure 3: Tracking of $x_{d}=\sin (0.1 \pi t)$ with $\tilde{\mathbf{x}}(0)=$ $[-0.7,-0.1]$.

Despite the external disturbance and the initial error, the AFSMC allows the Duffing oscillator to track the desired trajectory, and, as before, the undesirable chattering effect was not observed, Fig. 3(b).

The phase portrait associated with the last simulation is shown in Fig. 4(a). For comparison purposes, the phase portrait obtained with the conventional sliding modes is also presented, Fig. 4(b). Note that in both situations the steadystate tracking error remains on the convergence region $\Phi$, but the improved performance of the AFSMC can be easily observed.

\section{CONCLUDING REMARKS}

In this paper, an adaptive fuzzy sliding mode controller was developed to deal with uncertain single-input-single-output nonlinear systems. To enhance the tracking performance inside the boundary layer, the adopted strategy embedded an adaptive fuzzy algorithm within the sliding mode controller for uncertainty/disturbance compensation. The adoption of the switching variable $s$ in the premise of the rules, instead of the state variables, led to a smaller number of fuzzy sets and rules. Using Lyapunov's direct method and Barbalat's lemma, the boundedness of all closed-loop signals and other convergence properties were analytically proven. This result corrected flawed conclusions previously reached in the literature. To evaluate the control system performance, the pro-

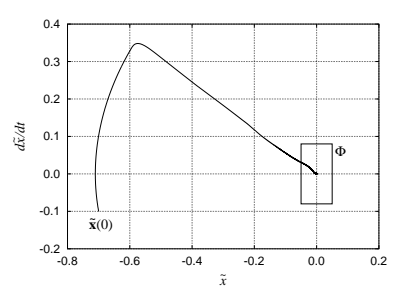

(a) With the proposed AFSMC.

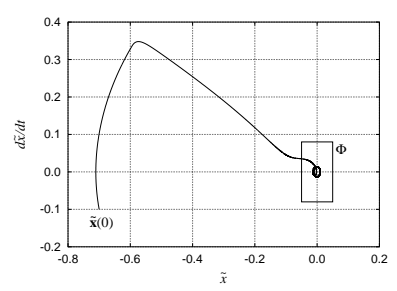

(b) With the conventional SMC.
Figure 4: Phase portrait of the trajectory tracking with $\tilde{\mathbf{x}}(0)=$ $[-0.7,-0.1]$. 
posed scheme was applied to the damped Duffing equation. Through numerical simulations, the improved performance over the conventional sliding mode controller was demonstrated.

\section{ACKNOWLEDGEMENTS}

The authors acknowledge the support of the State of Rio de Janeiro Research Foundation (FAPERJ). Moreover, the authors would like to thank Prof. Gilberto Oliveira Corrêa (LNCC) for his insightful comments and suggestions.

\section{REFERENCES}

Berstecher, R. G., Palm, R. and Unbehauen, H. D. (2001). An adaptive fuzzy sliding-mode controller, IEEE Transactions on Industrial Electronics 48(1): 18-31.

Bessa, W. M., De Paula, A. S. and Savi, M. A. (2007). An adaptive fuzzy sliding mode controller applied to a chaotic pendulum, PhysCon 2007 - Proceedings of the 3rd International IEEE Scientific Conference on Physics and Control, Potsdam, Germany.

Bessa, W. M., Dutra, M. S. and Kreuzer, E. (2007). Adaptive fuzzy sliding mode control of underwater robotic vehicles, DINAME 2007 - Proceedings of the XII International Symposium on Dynamic Problems of Mechanics, Ilhabela, Brazil.

Bessa, W. M., Dutra, M. S. and Kreuzer, E. (2008). Depth control of remotely operated underwater vehicles using an adaptive fuzzy sliding mode controller, Robotics and Autonomous Systems 56: 670-677.

Chai, T. and Tong, S. (1999). Fuzzy direct adaptive control for a class of nonlinear systems, Fuzzy Sets and Systems 103: 379-387.

Chang, W., Park, J. B., Joo, Y. H. and Chen, G. (2002). Design of robust fuzzy-model-based controller with sliding mode control for SISO nonlinear systems, Fuzzy Sets and Systems 125: 1-22.

Chen, H.-M., Renn, J.-C. and Su, J.-P. (2005). Sliding mode control with varying boundary layers for an electro-hydraulic position servo system, The International Journal of Advanced Manufacturing Technology 26: 117-123.

Cheng, C.-C. and Chien, S.-H. (2006). Adaptive sliding mode controller design based on T-S fuzzy system model, Automatica 42: 1005-1010.

De Paula, A. S., Savi, M. A. and Bessa, W. M. (2007). Chaos control in a nonlinear pendulum through an adaptive fuzzy sliding mode based approach, COBEM 2007 Proceedings of the 19th International Congress of Mechanical Engineering, Brasília, Brazil.

Filippov, A. F. (1988). Differential Equations with Discontinuous Right-hand Sides, Kluwer, Dordrecht.

Ha, Q. P., Nguyen, Q. H., Rye, D. C. and Durrant-Whyte, H. F. (2001). Fuzzy sliding mode controllers with applications, IEEE Transactions on Industrial Electronics 48(1): 38-46.

Jang, J.-S. R., Sun, C.-T. and Mizutani, E. (1997). Neuro Fuzzy and Soft Computing: A Computational Approach to Learning and Machine Intelligence, Prentice Hall, New Jersey.

Khalil, H. K. (2001). Nonlinear Systems, 3rd edn, Prentice Hall, New Jersey.

Kung, C.-C. and Chen, T.-H. (2005). Observer-based indirect adaptive fuzzy sliding mode control with state variable filters for unknown nonlinear dynamical systems, Fuzzy Sets and Systems 155: 292-308.

Liang, C.-Y. and Su, J.-P. (2003). A new approach to the design of a fuzzy sliding mode controller, Fuzzy Sets and Systems 139: 111-124.

Palm, R. (1994). Robust control by fuzzy sliding mode, $A u$ tomatica 30: 1429-1437.

Sharaf-Eldin, T., Dunnigan, M. W., Fletcher, J. E. and Williams, B. W. (1999). Nonlinear robust control of a vector-controlled synchronous reluctance machine, IEEE Transactions on Power Electronics 114(6): 11111121.

Slotine, J.-J. E. (1984). Sliding controller design for nonlinear systems, International Journal of Control 40(2): 421-434.

Slotine, J.-J. E. and Li, W. (1991). Applied Nonlinear Control, Prentice Hall, New Jersey.

Su, J.-P., Chen, T.-M. and Wang, C.-C. (2001). Adaptive fuzzy sliding mode control with ga-based reaching laws, Fuzzy Sets and Systems 120: 145-158.

Wang, J., Rad, A. B. and Chan, P. T. (2001). Indirect adaptive fuzzy sliding mode control: Part i and ii, Fuzzy Sets and Systems 122: 21-43.

Wang, L.-X. (1993). Stable adaptive fuzzy control of nonlinear systems, IEEE Transactions on Fuzzy Systems 1(2): 146-155. 
Wang, Q. and Su, C.-Y. (2006). Robust adaptive control of a class of nonlinear systems including actuator hysteresis with prandt-ishlinskii presentations, Automatica 42: 859-867.

Wang, X.-S., Su, C.-Y. and Hong, H. (2004). Robust adaptive control of a class of nonlinear systems with unknow dead-zone, Automatica 40: 407-413.

Wong, L. K., Leung, F. H. F. and Tam, P. K. S. (2001). A fuzzy sliding controller for nonlinear systems, IEEE Transactions on Industrial Electronics 48(1): 32-37.

Wu, T.-Z. and Juang, Y.-T. (2008). Adaptive fuzzy slidingmode controller of uncertain nonlinear systems, ISA Transactions 47: 279-285.

Yoo, B. and Ham, W. (1998). Adaptive fuzzy sliding mode control of nonlinear system, IEEE Transactions on Fuzzy Systems 6(2): 315-321.

Zhang, D. Q. and Panda, S. K. (1999). Chattering-free and fast-response sliding mode controller, IEE Proceedings - Control Theory and Applications 146(2): 171-177.

Zhang, T.-P. and Yi, Y. (2007). Adaptive fuzzy control for a class of MIMO nonlinear systems with unknown deadzones, Acta Automatica Sinica 33(1): 96-99. 